\title{
Editorial: Reframing the placebo effect. The importance of meaning
}

Rachel Perkins and Julie Repper

\section{Reframing the placebo effect. The importance of meaning}

The "placebo effect" has a long history in medicine. As long ago as 1572 French philosopher Michel de Montaigne observed that "there are men on whom the mere sight of medicine is operative" (cited in Hunter, 2007, p. 125). However, the first scientific demonstration of the placebo effect came in 1799 when physician John Haygarth set out to test one of the remedies available at the time - metal rods called "Perkins Tractors" - that were supposed to draw illness out of the body. He compared these with sham, wooden, rods that looked the same as "Perkins Tractors" and found them equally efficacious in reducing rheumatic pain (Goldacre, 2008, episode 1).

As Miller and Kaptchuk (2008) argue the term has been conceptualised in two different ways.

Firstly, the practice of giving people "inert" treatments (the "sugar pill" with which the placebo has become synonymous) with the aim of producing positive outcomes by satisfying their wishes. The word "placebo" derives from this practice: placebo is Latin for "I wish to please". This practice has been rightly criticised on ethical grounds because it involves deceiving the person and making a nonsense of informed consent.

Secondly, with the advent of scientific medicine, the "placebo effect" is seen as "noise" or "bias": a nuisance that must be "controlled for". New treatments are validated by demonstrating that they are superior to placebo in randomised, controlled trials conducted in "double-blind" conditions to establish their "real" therapeutic power. Healing has been equated with technological intervention and "the art of medicine, as reflected in the therapeutic potential of the clinical encounter, has been marginalised [...] Healing by means of technological intervention has eclipsed healing through the clinician-patient relationship" (Miller and Kaptchuk, 2008, p. 223).

Thus in an era of scientific psychiatry the concept of the placebo has been devalued and considered as lacking in effect, sham, dummy, inert. Interventions that pass the "randomised controlled trial" test are considered no better than "no treatment". However, this conclusion is misleading. The placebo may be substantially superior to no treatment (e.g. "waiting list" controls).

Increasingly it is being recognised that the placebo effect can enhance the effects of treatment. For example, Colloca et al. (2005) found that the effectiveness of analgesic medication was markedly reduced when it was given by a computer-controlled infusion pump without the person knowing when the drug is being given rather than openly by a doctor who described its pain-relieving qualities. It has also been demonstrated that the placebo effect does not require that the person receiving the intervention is deceived. KamHansen et al. (2014) conducted a study looking at whether the effect of the information provided influences both placebo and drug effects. It is noteworthy that this study shows that
Rachel Perkins is Senior Consultant at ImROC, London, UK. Julie Repper is Director at ImROC, London, UK. 
the placebo effect operates when the placebo is labelled as such: relative to no treatment, the placebo labelled "placebo" was superior. It was also noteworthy that those given the placebo labelled with the pain relief drug name, and those given the pain relief medication labelled as placebo were similar. Overall, the placebo, under each information condition, accounted for more than $50 \%$ of the drug effect.

In the field of mental health, Kirsch and Sapirstein (1998) showed that in antidepressant clinical trials for major depression, one quarter of the overall effect was due to the specific action of medication, one quarter to other factors (such as spontaneous remission) and one half to the real placebo effect - expectations of benefit. In a later analysis, Kirsch (2014) concludes that "[...] there is a strong therapeutic response to antidepressant medication. But the response to placebo is almost as strong" (Kirsch, 2014, 132).

This sort of finding has been associated with an increase in interest in the placebo effect and consideration not as a problem that must be controlled, but as an asset that must be maximised and explored. As Hunter (2007) argues, "[...] there has been a tendency to regard the placebo effect as background "noise" that must be subtracted from the results of a trial, rather than a positive effect that could be exploited clinically" (Hunter, 2007, 126).

Some, such as Bendetti et al. (2011), have investigated the neuorobiological basis of the placebo effect. They describe "not a single placebo effect but many placebo effects with different mechanisms and in different systems, medical conditions and therapeutic interventions. For example, brain mechanisms of expectation, anxiety and reward are all involved as well as a variety of learning phenomena, such as Pavlovian conditioning, cognitive and social learning" (Bendetti et al., 2011, 339).

However, perhaps most interest in the placebo effect appears to have focussed on the importance of the client-clinician interaction. Ben Goldacre says that "The benefits of a treatment can be enhanced or detracted based on how you treat the patient" and talks of "[...] pivotal role of ceremony and the doctor in the healing process" (Goldacre, 2008, episode 2). Benedetti et al. (2011) argue that the placebo effect is "[. . . basically a psychosocial context effect [...] different social stimuli, such as the words and rituals of the therapeutic act, may change the chemistry and circuitry of the patient's brain" (Benedetti et al., 2011, 339).

Miller and Kaptchuk (2008) argue that the placebo concept needs to be reconceptualised as "contextual healing": "Healing resulting from the clinical encounter consists of a causal connection between clinician-patient interaction (or a particular component of the interaction) and improvement in the condition of the patient" (Miller and Kaptchuk, 2008, 224). They postulate that there may be a number of factors involved in contextual healing, including the environment of the clinical setting, communication of clinicians (both cognitive and affective) and the rituals of administering treatment:

"Instead of focusing exclusively on the therapeutic power of medical technology and thereby ignoring or dismissing context, we should see the context of the clinical encounter as a potential enhancer, and in some cases the primary vehicle, of therapeutic benefit."

However, we would argue that this reconceptualization from placebo to contextual healing is important, but does not go far enough: a broader understanding of "context" is needed to understand the large individual and cultural variations in contextual healing (placebo effect) of which Hunter (2007) speaks.

As Hunter (2007) argues, the placebo effect (or Miller and Kaptchuk's, 2008, "contextual healing") requires that the patient believes in the treatment. Too often in the mental health arena this is not the case. There are many who do not believe in their 
doctor's construction of their "illness" and therefore do not have positive expectations of the treatments that they are offered.

We would argue that meaning is a critical part of the context that must be considered.

The essence of the placebo or contextual healing effect is that if we think that some intervention or support will be beneficial then it will have a positive impact, even if the research base of scientific psychiatry has not demonstrated its effectiveness. It is reasonable to assume that our beliefs about the nature of our mental health challenges and their meaning for us in large part determine our expectations of different interventions and our experience of the clinical encounter.

There is no one way of understanding mental health challenges. Different clinicians adopt a range of different organic, social, psychological, political, psychoanalytic and systemic theories and their associated interventions. Similarly, Jacobson (1993) showed that people with mental health challenges adopt an equally large range of ways of understanding what had happened: biological models, biological and environmental models (an innate vulnerability combined with stressful life events), abuse/trauma models, spiritual or philosophical models (a spiritual crisis) political models (marginalisation, exclusion, racism, sexism, denial of rights, etc., lie at the root of the person's problem) and "spirit breaking" models (difficulties arising from the dehumanising impact of the mental health system itself). It is not unreasonable to assume that these different models lead to different expectations of interventions.

It is perhaps unsurprising that within the diverse communities of a multicultural society, there are large individual and cultural differences in the understanding of mental health challenges and expectations of mental health services (both positive and negative). This diversity of meanings lies at the root of the large individual and cultural variations in the placebo effect/ contextual healing.

As Benedetti et al. (2011) argue: "Placebos [...] are made up of words and rituals, symbols and meanings [...]". For someone holding a biological model of mental distress, the symbols and meanings of the clinical encounter with a psychiatrist may have a positive placebo/contextual healing effect, but for someone holding one of the myriad spiritual models these same symbols and meanings may have the opposite impact. For a person holding such beliefs the meaning of the words, rituals and symbols of their faith may have a greater contextual healing (placebo) effect, whereas for someone holding psychological understandings the words and rituals of their chose psychological intervention may produce the largest contextual healing effect. Concordance or compatibility of mental health beliefs between individual and clinician may therefore be of the essence.

Within mental health services, it is not uncommon for clinicians to spend time explaining what is wrong with the person from within the practitioner's frame of reference and offer treatments in line with this. If the person fails to accept the professional construction of their challenges then, too often, they are deemed to "lack insight" (Repper and Perkins, 2003). We have no "magic bullets" in terms of treatments and interventions, and if beliefs and meaning are important, then this approach is unlikely to maximise the powerful contextual healing (placebo) effect. As Spaniol, Gagne and Koehler have argued, such an approach has "left many people with mental illness feeling devalued and ignored and has resulted in mistrust and alienation" (Spaniol et al., 1997, 7).

"A recovery vision does not require a person to adopt any particular model or understanding of his/her difficulties. Practitioners should not see their role as persuading the person of the 'correct' way to understand their situation. Veracity is not the key issue as far as recovery is concerned. The critical thing is that the person finds a way of understanding what has happened that satisfies two criteria: it must both make sense to him/her and offer the possibility of actively moving forward and rebuilding his/her life." (Repper and Perkins, 2003, 102). 
To maximise the powerful contextual healing effect, it is important to move beyond ideas about "correct" and "incorrect" beliefs and accept that everyone's construction of reality is different. As Romme and Escher (1993) conclude,

"It is of little value to try to force a particular explanation upon someone; it should be recognised that other frames of reference may offer additional explanations and in any case are likely to be sought out by the patient regardless of the therapist's personal insistence" (Romme and Escher, 1993).

Similarly, we also need to move beyond assessing a person's suitability for different treatments, therapies and supports available and prescribing the one the professional considers optimal. If contextual healing is to be maximised, it may be preferable to outline the different options available (including things other than therapy or medication that some people have found helpful) and allowing the person to choose which one, or combination of possibilities, they would prefer. In this context, it is worth noting that Khan et al. (2012) performed a meta-analysis of treatments for depression (including antidepressants, psychotherapy, a combination of the two, and "alternative" treatments including acupuncture and physical exercise) and found no significant differences between these or within different genres of psychotherapy.

Perhaps then, the challenge of maximising contextual healing is to assist people to develop their own "personal medicine": "[. . .] self-initiated, non-pharmaceutical self-care activities that served to decrease symptoms, avoid undesirable outcomes such as hospitalisation, and improve mood, thoughts, behaviors and overall sense of wellbeing" (Deegan, 2005, 31).

"Over the years I have learned a variety of ways of helping myself. Sometimes I use medications, therapy, self-help and mutual support groups, friends, my relationship with God, work, exercise, spending time in nature - all these measures help me remain whole and healthy [...]" (Deegan, 1993, 10).

Interestingly, Deegan's (2005) research indicated that few people had told their health-care providers about their "personal medicine", and few health-care providers had enquired. At the same time, she found that where psychiatric medication interfered with a person's personal medicine, people often stopped taking it. She found two main categories of personal medicine: specific self-care strategies and activities that gave meaning and purpose in life, both of which helped people to gain "[...] the personal agency, will, vision, hope, fortitude, courage, imagination, commitment and resilience [... ]" (Deegan, 2005, 31) necessary for recovery.

Perhaps, when treatment plans support the individual's personal medicine, then contextual healing may be maximised?

\section{References}

Benedetti, F., Carlino, E. and Pollo, A. (2011), "How placebos change the patient's brain", Neuropsychopharmacology, Vol. 36 No. 1, pp. 339-354.

Deegan, P. (1993), "Recovering our sense of value after being labeled mentally ill"

Deegan, P. (2005), "The importance of personal medicine: a qualitative study of resilience in people with psychiatric disabilities”, Scandinavian Journal of Public Health, Vol. 33 No. 66_suppl, pp. 29-35.

Goldacre, B. (2008), "Placebo. BBC radio programme", August 2008 Episode 1 available at: www.bbc. co.uk/programmes/b00d43fzEpisode2 www.bbc.co.uk/programmes/b00d0f3p

Hunter, P. (2007), "A question of faith”, EMBO Reports, Vol. 8 No. 2, pp. 125-128.

Kam-Hansen, S., Jakubowski, M., Kelley, J.M., Kirsch, I., Hoaglin, D.C., Kaptchuk, T.J. and Burnstein, R. (2014), "Altered placebo and drug labelling changes and outcome of episodic migraine attacks", Science Translational Medicine, Vol. 6 No. 218, pp. 1-7.

Khan, A., Faucett, J., Lichtenberg, P., Kirsch, I. and Brown, W.A. (2012), "A systematic review of comparative efficacy of treatments and controls for depression", PLoS One, Vol. 7 No. 7, p. e41778, doi: 10.1371/journal. pone.0041778. Epub 2012 Jul 30. PMID: 22860015; PMCID: PMC3408478. 
Kirsch, I. (2014), "Antidepressants and the placebo effect”, Zeitschrift Für Psychologie, Vol. 222 No. 3, pp. 128-134.

Kirsch, I. and Sapirstein, G. (1998), "Listening to Prozac but hearing placebo: a Meta-analysis of antidepressant medication", Prevention \& Treatment, Vol. 1 No. 2, doi: 10.1037/1522-3736.1.1.12a. Article $2 a$

Miller, F.G. and Kaptchuk, T.J. (2008), "The power of context: reconceptualising the placebo effect", Journal of the Royal Society of Medicine, Vol. 101 No. 5, pp. 222-225.

Repper, J. and Perkins, R. (2003), Social Inclusion and Recovery. A Model for Mental Health Practice, Balliere Tindall, London.

Romme, M. and Escher, S. (1993), Accepting Voices, Mind Publications, London.

\section{Further reading}

Journal of Psychosocial Nursing and Mental Health Services, Vol. 31 No. 4, pp. 7-11.

For instructions on how to order reprints of this article, please visit our website: www.emeraldgrouppublishing.com/licensing/reprints.htm

Or contact us for further details: permissions@emeraldinsight.com 